\title{
Single Nucleotide Polymorphisms (SNPs) Distant from Xenobiotic Response Elements Can Modulate Aryl Hydrocarbon Receptor Function: SNP-Dependent CYP1A1 Induction ${ }^{\mathbb{S}}$
}

\author{
Duan Liu, Sisi Qin, Balmiki Ray, ${ }^{1}$ Krishna R. Kalari, Liewei Wang, and Richard M. Weinshilboum \\ Division of Clinical Pharmacology, Department of Molecular Pharmacology and Experimental Therapeutics (D.L., S.Q., B.R., L.W., \\ R.M.W.) and Division of Biomedical Statistics and Informatics, Department of Health Sciences Research (K.R.K.), Mayo Clinic, \\ Rochester, Minnesota
}

Received April 22, 2018; accepted June 28, 2018

\begin{abstract}
CYP1A1 expression can be upregulated by the ligand-activated aryl hydrocarbon receptor (AHR). Based on prior observations with estrogen receptors and estrogen response elements, we tested the hypothesis that single-nucleotide polymorphisms (SNPs) mapping hundreds of base pairs (bp) from xenobiotic response elements (XREs) might influence AHR binding and subsequent gene expression. Specifically, we analyzed DNA sequences $5 \mathrm{~kb}$ upstream and downstream of the CYP1A1 gene for putative XREs. SNPs located \pm 500 bp of these putative XREs were studied using a genomic datarich human lymphoblastoid cell line (LCL) model system. CYP1A1 mRNA levels were determined after treatment with varying concentrations of 3-methylcholanthrene (3MC). The rs2470893 (-1694G>A) SNP, located 196 bp from an XRE in the CYP1A1 promoter, was associated with 2-fold variation in AHR-XRE binding in a SNP-dependent
\end{abstract}

fashion. LCLs with the AA genotype displayed significantly higher AHR-XRE binding and CYP1A1 mRNA expression after 3MC treatment than did those with the GG genotype. Electrophoretic mobility shift assay (EMSA) showed that oligonucleotides with the AA genotype displayed higher LCL nuclear extract binding after 3MC treatment than did those with the GG genotype, and mass spectrometric analysis of EMSA protein-DNA complex bands identified three candidate proteins, two of which were co-immunoprecipitated with AHR. In conclusion, we have demonstrated that the rs2470893 SNP, which maps 196 bp from a CYP1A1 promoter XRE, is associated with variations in 3MC-dependent AHR binding and CYP1A1 expression. Similar "distant SNP effects" on AHR binding to an XRE motif and subsequent gene expression might occur for additional AHR-regulated genes.

\section{Introduction}

The aryl hydrocarbon receptor (AHR) is a ligand-activated transcription factor that is best known for its role in response to environmental toxins (Denison and Nagy, 2003; Murray et al., 2014). Once activated, AHR forms a heterodimer with the AHR nuclear transporter (ARNT) that binds to specific DNA sequence motifs, xenobiotic response elements (XREs), resulting in the regulation of gene transcription (Mimura and Fujii-Kuriyama, 2003). Although a structurally diverse group of chemical compounds are AHR ligands, the best characterized high-affinity ligands are hydrophobic agents, such as halogenated aromatic hydrocarbons and nonhalogenated polycyclic aromatic hydrocarbons (Denison and Nagy, 2003). A prototypic gene that is induced by AHR activation is CYP1A1, which encodes cytochrome

This work was supported by the National Institutes of Health National Institute of General Medical Sciences (NIGMS) [Grants U19 GM61388, R01 GM28157, and R01 GM125633].

${ }^{1}$ Current affiliation: Assurex Health Inc., Mason, Ohio.

https://doi.org/10.1124/dmd.118.082164.

SThis article has supplemental material available at dmd.aspetjournals.org.
P450 1A1, an enzyme that metabolizes xenobiotics and environmental toxins, many of which are AHR ligands (Denison and Nagy, 2003). Several XRE motifs have been identified across the CYP1Al gene (Denison et al., 1988; Lo and Matthews, 2012).

CYP1A1 expression can vary widely, which theoretically might contribute to variation in cancer risk after carcinogen exposure (e.g., lung cancer risk in smokers) (Rannug et al., 1995; Stucker et al., 2000). As a result, many studies have been performed searching for possible associations of CYP1A1 single nucleotide polymorphisms (SNPs) with the induction of CYP1A1 expression and carcinogenesis risk; however, the results have often been contradictory — with some studies, especially those performed in East Asia, reporting a correlation of CYP1A1 genetic polymorphisms with CYP1A1 induction and/or cancer risk (Kawajiri et al., 1990; Kiyohara et al., 1998), whereas others have failed to replicate those observations (Inoue et al., 2000; Anttila et al., 2001; Smith et al., 2001). Most of those studies focused on two common CYP1A1 SNPs: rs4646903, also known as $C Y P 1 A 1 * 2 A$, which maps $3^{\prime}$ of CYP1A1 (Spurr et al., 1987), and rs1048943, a nonsynonymous SNP that results in an Ile462Val substitution without change in enzyme activity (Zhang et al., 1996; Persson et al., 1997). Both of these SNPs have higher minor allele frequencies in East Asian populations than

ABBREVIATIONS: 3MC, 3-methylcholanthrene; AHR, aryl hydrocarbon receptor; ARNT, AHR nuclear transporter; ChIP, chromatin immunoprecipitation; Co-IP, coimmunoprecipitation; CYP1A1, cytochrome P450 1A1; DMSO, dimethyl sulfoxide; EMSA, electrophoretic mobility shift assay; ERE, estrogen response element; FIMO, Find Individual Motif Occurrences; GWAS, genome-wide association study; KD, knockdown/knocked down; LCL, lymphoblastoid cell line; MS, mass spectrometry; SNP, single nucleotide polymorphism; qRT-PCR, quantitative reverse transcription polymerase chain reaction; V, variant; WT, wild-type; XRE, xenobiotic response element. 
in Europeans, which might explain the contradictory results of attempts to associate $C Y P 1 A 1$ genetic polymorphisms with CYP1A1 induction and/or cancer risk; however, exactly how those two SNPs might alter CYP1A1 activity remains unclear. Except for one AHR SNP (Smart and Daly, 2000) that was reported to influence CYP1A1 induction, no SNPs have been associated with the induction of expression of this gene. As a result, the molecular basis for individual variation in the inducibility of CYP1A1 is not completely understood.

Recently, during studies of estrogen receptor $\alpha$, we observed that SNPs at a distance (i.e., hundreds of base pairs) from estrogen response elements (EREs) could strongly influence both ER binding to those EREs and subsequent gene expression (Ingle et al., 2013, 2016; Ho et al., 2016; Liu et al., 2017). To determine whether this type of phenomenon might also occur with other nuclear receptors, we set out in the present study to test the hypothesis that SNPs at a significant distance from XREs might modulate AHR-XRE binding and influence the transcription of AHR-regulated genes such as CYPIA1, a process that might, in part, explain individual variation in CYP1A1 inducibility. $\mathrm{ER} \alpha$ acts in a fashion very similar to AHR. Once activated by estrogen binding, $\mathrm{ER} \alpha$ forms a homodimer and binds to specific DNA sequence motifs in EREs, thus regulating gene expression. For example, a previous genome-wide association study (GWAS) for response to tamoxifen breast cancer prevention therapy identified SNP signals in intron two of the ZNF423 gene (Ingle et al., 2013). Functional genomic studies demonstrated that the ZNF423 rs9940645 SNP, which maps 200 bp away from an ERE cluster, could influence ER $\alpha$-ERE binding and ZNF423 transcription (Ingle et al., 2013). Furthermore, subsequent studies identified calmodulin-like protein 3 as a co-regulator of $\operatorname{ER} \alpha$ that "sensed" genotypes for the ZNF423 intron two SNP (Qin et al., 2017).

In the present study, we aimed to determine whether SNPs at a distance from XREs might modulate AHR-XRE binding and influence downstream gene transcription in a fashion similar to the effect of the rs9940645 SNP in ZNF423 on ER $\alpha$ binding to EREs by studying SNPs and XREs in and near CYP1A1. As described subsequently, we identified a SNP, rs2470893, that maps 5' - of CYP1A1 that can influence both AHR binding to an XRE located 196 bp distant from the SNP and subsequent CYP1A1 transcription induced by the AHR agonist, 3-methylcholanthrene (3MC) (Riddick et al., 1994). We also identified possible AHR interacting proteins that might help to explain the mechanism responsible for this process.

\section{Materials and Methods}

Identification of Putative XREs. A motif scanning tool, Find Individual Motif Occurrences (FIMO) (http://meme-suite.org/tools/fimo), was applied to identify sequences matched to known XRE motifs (Denison et al., 1988; Sogawa et al., 2004). DNA sequences of the two known XRE motifs were used as templates for putative XRE scanning, the XRE-I motif with a sequence of CACGCNA (Denison et al., 1988), and the XRE-II motif, with a sequence of "CATGN ${ }_{6}$ C[T/A]TG" (Sogawa et al., 2004). Based on known transcriptional activity sites for the CYP1A1 gene included in ENCODE (https:// genome.ucsc.edu/ENCODE), DNA sequences located $5 \mathrm{~kb}$ upstream and downstream of the CYP1Al gene, as well as across the gene, were analyzed using FIMO to identify putative XREs.

Cell Culture. The Human Variation Panel of lymphoblastoid cell lines (LCLs) was obtained from the Coriell Institute (Camden, NJ). The DNA from these 300 LCLs had been genotyped in the Coriell Institute using the Affymetrix Human SNP Array 6.0 (Affymetrix, Santa Clara, CA) and in our laboratory using Illumina HumanHap 550K and HumanExon 510S-Duo BeadChips (Illumina, San Diego, CA) to obtain genome-wide SNP data. Imputation was then performed using 1000 Genomes data. In addition, we generated gene expression data for all of the LCLs by using Affymetrix U133 2.0 Plus GeneChip expression arrays.
These genotype and gene expression data were deposited in the National Center for Biotechnology Information Gene Expression Omnibus (GEO accession: GSE23120) (Niu et al., 2010). This LCL model system was previously used successfully to preform many studies of SNP-dependent gene transcription (Ingle et al., 2013; Ho et al., 2016; Liu et al., 2017). The LCLs were cultured in RPMI 1640 media containing $15 \%$ fetal bovine serum.

Undifferentiated HepaRG human hepatic cells (HPR101) were obtained from Biopredict (Rennes, France) and were cultured and differentiated into functional hepatocyte-like cells according to the manufacture's protocol. HPR101 cells were grown in William's E media (Gibco, Grand Island, NY) supplemented with $1 \times$ GlutaMAX (Gibco) and 10\% HepaRG growth medium supplement (Biopredict) for 10-14 days until confluent. The cells were then switched into HepaRG Differentiation media for 2 weeks. Differentiated HepaRG cells were used to preform transfection studies.

Human SK-N-BE(2) neuroblastoma and U-87 MG glioblastoma cell lines were obtained from the American Type Culture Collection (ATCC; Manassas, VA). Original stocks of the cells were stored in liquid nitrogen and had not been passaged. Both these cell lines were cultured in Eagle's minimum essential medium with $10 \%$ fetal bovine serum.

3-Methylcholanthrene (3MC) Treatment. A "prototypic" AHR ligand, 3MC (Sigma, St. Louis, MI), was used to treat LCLs, HepaRG, and U87 MG cells to activate AHR signaling pathways. Control cells were treated with dimethyl sulfoxide (DMSO), which had been used to dissolve the 3MC. The DMSO final concentration in the cell culture media was $0.1 \%(\mathrm{v}: \mathrm{v})$ for all of the control treatments. For CYP1A1 mRNA quantification, LCLs were treated with $3 \mathrm{MC}$ at a series of concentrations ranging from $1 \mathrm{E}-06$ to $10 \mu \mathrm{M}$ for 24 hours and were then harvested for total RNA extraction. For chromatin immunoprecipitation (ChIP) assays, cells were incubated with $0.1 \mu \mathrm{M} 3 \mathrm{MC}$ for 2 hours and were then fixed by exposure to $1 \%$ formaldehyde. For reporter gene assays, cells were treated with $0.1 \mu \mathrm{M} 3 \mathrm{MC}$ for 24 hours before harvest for use in subsequent experiments.

mRNA Quantification. mRNA levels were determined by quantitative reverse transcription polymerase chain reaction (qRT-PCR) using the Power SYBR Green RNA-to-CT 1-Step kit (Applied Biosystems Inc., Foster City, CA). Total RNA was extracted from cells with the Quick-RNA MiniPrep Plus kit (Zymo Research, Irvine, CA). A total of 100 ng of total RNA was used in each reaction. Glyceraldehyde 3-phosphate dehydrogenase (GAPDH) mRNA expression was measured for use as an internal control. Specific primers for CYP1A1, AHR, GAPDH mRNA, as well as other genes, were purchased from Integrated DNA Technologies (Coralville, IA). Primer sequences for those genes are listed in Supplemental Table 1.

ChIP Assay. ChIP assays were conducted for LCLs using the EpiTect ChIP OneDay Kit (QIAGEN, Germantown, MD). In brief, approximately $5 \times 10^{6}$ cells were treated with $0.1 \mu \mathrm{M} 3 \mathrm{MC}$ for 2 hours. The same number of cells that had been treated with DMSO was used as a control. After the cells were cross-linked and harvested, $400 \mu 1$ of lysis buffer was added to lyse the cells. DNA was sheared by sonication into 200- to 1000-bp fragments, with an average size of $500 \mathrm{bp}$. Aliquots of $100 \mu \mathrm{l}$ of sheared chromatin/DNA was used for immunoprecipitation with an AHR antibody (cat no. 83200S; Cell Signaling, Danvers, MA) or IgG (cat. no. 2729S; Cell Signaling). Two microliters of purified ChIP DNA was added to a final 10- $\mu \mathrm{l} \mathrm{qPCR}$ reaction mixture for the quantification of putative XRE sequences. SYBR Green ROX qPCR Mastermix (QIAGEN, Hilden, Germany) was used to amplify ChIP DNA. The qPCR primers used to amplify specific DNA fragments, including putative XREs and SNPs, are listed in Supplemental Table 2. Functional XREs were defined based on greater than 2 -fold enrichment compared with DMSO-treated cells after exposure to AHR antibody in 3MC-treated cells.

Transient Transfection. LCLs were transfected with DNA plasmids for reporter gene assay or with siRNA for gene knockdown (KD) by electroporation using the Amaxa Cell Line Nucleofector Solution V Kit (Lonza, Cologne, Germany). Specifically, LCLs were maintained at $1 \times 10^{6}$ cells $/ \mathrm{ml}$ for 2 days before use. For each transfection, $1 \times 10^{6}$ cells were mixed with $2 \mu \mathrm{g}$ of plasmid DNA or 100 pmol of siRNA in $100 \mu 1$ of Nucleofector Solution V and incubated in cuvettes for 5 minutes without air bubbles. Sample suspensions in cuvettes were then "pulsed" on a Nucleofector II Device (Lonza) with program X-001. After the addition to the cuvette of $500 \mu 1$ of pre-equilibrated culture media, samples were gently transferred to culture plates and incubated at $37^{\circ} \mathrm{C}$ overnight. Cells were then treated with $3 \mathrm{MC}$ as described already before use. SK-N-BE(2) 
cells were transfected in the same fashion as LCLs using the Nucleofector II Device but with the X-005 program. HepaRG and U87 MG cells were transfected with reporter gene constructs and/or siRNA by using the Lipofectamine 3000 Transfection Reagent (ThermoFisher, Grand Island, NY). Twenty-four hours after transfection, cell culture media were replaced with fresh media containing $0.1 \mu \mathrm{M} 3 \mathrm{MC}$ for an additional 24 hours. The cells were then harvested for the extraction of total RNA and for luciferase activity.

Reporter Gene Assay. To determine the effects of SNP on CYP1A1 transcription, an approximately $2.7-\mathrm{kb}$ DNA segment that included sequence upstream (5') and exon 1 of CYP1A1 were subcloned upstream of the luc2 gene (encoding firefly luciferase) in the pGL4-Basic vector (Promega, Madison, WI). DNA sequences of the primers used for subcloning were $5^{\prime}$-ttgcCTCGAGTAATTGGACGGAGCAAAAGG-3' (forward primer with the XhoI restriction enzyme site underlined) and 5'-gcgAAGCTTAGAAAGGGCAAGCCAGAAGT-3' (reverse primer with the HindIII restriction enzyme site underlined). Genomic DNA from LCLs with known genotypes for the SNP of interest was used as a template for PCR reactions designed to amplify $2.7-\mathrm{kb}$ DNA fragments containing wild-type (WT) or variant (V) SNP alleles. After confirmation of the sequence by DNA sequencing, reporter gene constructs containing WT or V SNP genotypes were transfected into cells as described already. A pRL-CMV vector (Promega) that expresses renilla luciferase was cotransfected as an internal control. After 3MC treatment, the cells were lysed, and luciferase activity was determined using the Dual-Luciferase Reporter assay system (Promega).

EMSA and MS. To determine whether the DNA sequence containing the SNP of interest might bind to nuclear proteins, EMSA was performed with nuclear extracts from LCLs after treatment with 3MC or DMSO. DNA oligonucleotides (Oligoes) 21 nucleotides long with the SNP in the middle were synthesized by Integrated DNA Technologies. The DNA sequences of oligonucleotides for the SNP of interest are listed in Supplemental Table 3. A similar set of oligonucleotides was synthesized with a biotin label at the $5^{\prime}$-end. Double-stranded DNA fragments were obtained by annealing "sense" and "antisense" oligonucleotides, and those dsDNA fragments were incubated with LCL nuclear extracts by using the LightShift EMSA Optimization \& Control Kit (Thermo Scientific, Rockford, IL). Nuclear extracts from LCLs treated with 3MC were prepared by using the Nuclear Extract Kit (Active Motif, Carlsbad, CA). After incubation, the dsDNA-protein complexes were separated in parallel using two 5\% Mini-PROTEAN TBE Precast Gels (Bio-Rad, Hercules, CA). After separation, one gel was used to transfer dsDNA fragments to Biodyne B precut modified nylon membranes, $0.45 \mu \mathrm{m}$ (Thermo Scientific). Biotin-labeled dsDNA on the membranes was then visualized using the Chemiluminescent Nucleic Acid Detection module (Thermo Scientific). The other gel was stained using the Pierce Silver Stain kit (Thermo Scientific). EMSA bands were then excised from the gel and sent for mass spectrometry (MS) analysis. Sample processing and MS assays were performed by the Taplin Biologic Mass Spectrometry Facility at Harvard Medical School.

Coimmunoprecipitation. LCLs with homozygous variant genotypes $(V / V)$ for the rs2470893 SNP $(n=3)$ were treated with 3MC for 24 hours. Similar amount of each LCL, approximately $10^{7}$ cells, were mixed for total protein extraction using NETN buffer (100 mM NaCl, $20 \mathrm{mM}$ Tris-Cl (pH 8.0), $0.5 \mathrm{mM}$ EDTA, and $0.5 \%$ Nonidet P-40) with protease and phosphatase inhibitor cocktails (Roche, Indianapolis, IN). Coimmunoprecipitation (Co-IP) was then performed as described previously (Yu et al., 2018). Antibodies against AHR, RCC1, NAP1L4, and DDX39B or control IgG were used to perform Co-IP and Western blot analysis. Reverse Co-IP was performed using antibodies directed against RCC1, NAP1L4, and UAP56, respectively. AHR antibody was used for the Western blot analysis. Information about the antibodies used in the Co-IP and Western blot analyses is listed in Supplemental Table 4.

Statistical Analysis. Data analyses were performed, and graphs were plotted using GraphPad Prism (GraphPad Software, La Jolla, CA). Statistical comparisons were made using Student's $t$ test or one-way analysis of variance.

\section{Results}

Identification of Putative XREs and SNP Selection. To test the hypothesis that SNPs distant from XREs in the CYPlAl gene might influence its induction, we first identified putative XREs near and across the gene by scanning DNA sequences across and $5 \mathrm{~kb}$ upstream and downstream of the CYPlAl gene for sequences that match known XRE sequence motifs. Fifteen putative XREs were identified, of which seven matched XRE-I and eight matched XRE-II sequences (see Supplemental Table 5). We failed to identify any SNP that either disrupted or created XRE sequences. Since our purpose was to determine the possible function of SNPs at a distance from XREs, SNPs located \pm 500 bp of these putative XREs were included in the studies described subsequently (Supplemental Table 5). We chose to study SNPs that mapped $\pm 500 \mathrm{bp}$ of an XRE because those SNPs could be studied with the greatest accuracy regarding AHR-XRE binding by the application of ChIPqPCR assay with the amplification of both the XRE and SNP sequences (Ingle et al., 2013; Liu et al., 2017). Specifically, during ChIP assays, the chromatin is often sheared into fragments 200-1000 bp long, with an average size of 500 bp (Nelson et al., 2006; Dahl and Collas, 2008; Saleh et al., 2008; Carey et al., 2009). Chromatin fragments 400-500 bp long have proven to be most suitable for PCR-based ChIP assays since they cover two to three nucleosomes (Dahl and Collas, 2008). This length of the chromatin fragments limited our SNP selection since only fragments with AHR bound to the XRE would be precipitated by the AHR antibody, and only SNP(s) within the same chromatin fragment would be detected. SNPs located farther from the XRE being studied would not be precipitated. To determine possible associations of SNPs with CYP1A1 induction, we used a human lymphoblastoid cell line (LCL) model system consisting of cells from 300 individuals of three different ethnic groups, for which genome-wide SNP genotype and mRNA expression data had been generated in our laboratory (Niu et al., 2010). Specifically, LCLs with WT or homozygous variant genotypes for the SNPs of interest were treated with an AHR agonist (3MC), followed by determination of CYP1A1 mRNA levels. We excluded SNPs having a minor allele frequency $<0.10$ because it would be practically difficult to study those SNPs because of the limited number of LCLs homozygous for variant SNP genotypes. Four SNPs within the area studied met these criteria, including the CYPlAl MspI variant, and three other SNPs that mapped 5' of the CYP1A1 gene (Table 1).

SNP-Dependent CYP1A1 Induction. To determine whether any of these four SNPs (Table 1) might be associated with variation in CYP1A1 induction, we chose 14 LCLs based on their genotypes for these four SNPs (Supplemental Table 6). Since a previous GWAS that we had performed for symptom severity of major depressive disorder had shown that the AHR rs17137566 SNP was an eQTL in European populations (Liu et al., 2018), and a previous study had reported that the $A H R$ rs2066853 SNP appeared to influence CYP1A1 induction (Smart and Daly, 2000), we excluded LCLs that were homozygous variant genotype for those two $A H R$ SNPs to avoid significant differences in baseline AHR activities in the 14 LCLs that we chose to study. These 14 LCLs were then treated for 24 hours with $3 \mathrm{MC}$ at concentrations that ranged from 1E-06 to $10 \mu \mathrm{M}$. CYP1A1 mRNA levels were then determined by qRT-PCR. We found that the induction of CYP1A1 mRNA differed by genotype for the rs 2470893 SNP (Fig. 1). This SNP was located $1570 \mathrm{bp}$ from the 5'-end of the CYP1A1 transcription-start site, $196 \mathrm{bp}$ from a putative XRE (Fig. 1A). CYP1A1 induction in LCLs with homozygous variant genotypes $(V / V)$ for the rs2470893 SNP was significantly higher than in LCLs homozygous for the WT genotype (W/W) (Fig. 1B). Genotypes for the three other SNPs listed in Table 1, either individually or in combination, were not associated with differences in CYP1A1 induction after $3 \mathrm{MC}$ treatment (data not shown). To determine whether this rs2470893 SNP-dependent difference in CYP1A1 induction might be caused by differences in AHR binding to the nearby putative XRE sequence, ChIP assays with anti-AHR antibody were performed using LCLs with $W / W$ and $V / V$ genotypes after 2 hours of $3 \mathrm{MC}$ treatment. A significant increase in enrichment of SNP/XRE DNA fragments was observed in LCLs with the $V / V$ genotype but not in those with the $W / W$ genotype (Fig. 1C). Except for the rs2470893 SNP, we did not observe a 
TABLE 1

Common single nucleotide polymorphisms (SNPs) that mapped $\pm 500 \mathrm{bp}$ of xenobiotic response elements (XREs) across or $5 \mathrm{~kb}$ upstream and downstream of the CYP1Al gene

\begin{tabular}{|c|c|c|c|c|c|c|c|c|c|c|c|c|}
\hline \multirow{2}{*}{ SNP rsID } & \multirow{2}{*}{ Common Allele } & \multirow{2}{*}{ Minor Allele } & \multicolumn{5}{|c|}{$\begin{array}{l}\text { Minor Allele Frequency } \\
\text { (1000 Genomes Data) }\end{array}$} & \multirow{2}{*}{ Putative XRE Sequences } & \multirow{2}{*}{ Motif Type $^{a}$} & \multirow{2}{*}{$P$ Value $^{b}$} & \multirow{2}{*}{$\begin{array}{l}\text { Distance (bp) } \\
\text { XRE to Gene }\end{array}$} & \multirow{2}{*}{$\begin{array}{l}\text { Distance (bp) } \\
\text { SNP to XRE }\end{array}$} \\
\hline & & & EUR & EAS & AFR & AMR & SAS & & & & & \\
\hline rs17861109 & $\mathrm{C}$ & A & 0.107 & 0.427 & 0.371 & 0.419 & 0.338 & CACGCCA & XRE-I & $8.78 \mathrm{E}-05$ & -2106 & 259 \\
\hline rs2470893 & G & A & 0.285 & 0.000 & 0.016 & 0.138 & 0.050 & CAGGGAAGGCCTTG & XRE-II & $8.38 \mathrm{E}-05$ & -1766 & 196 \\
\hline rs3826041 & A & $\mathrm{C}$ & 0.154 & 0.538 & 0.570 & 0.470 & 0.363 & CACGCGA & XRE-I & $4.39 \mathrm{E}-05$ & -1045 & 33 \\
\hline rs4646903 (MspI) & $\mathrm{T}$ & $\mathrm{C}$ & 0.107 & 0.430 & 0.235 & 0.411 & 0.340 & CACGCCA & XRE-I & $8.78 \mathrm{E}-05$ & 294 & 53 \\
\hline
\end{tabular}

AFR, African, $n=1322$; AMR, admixed American, $n=694$; EAS, East Asian, $n=1008$; EUR, European, $n=1006$; SAS, South Asian, $n=978$. Base on 1000 Genomes data, the rs2470893 SNP

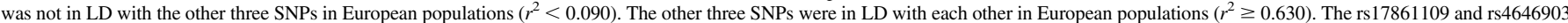
SNPs were tightly linked $\left(r^{2}=1.000\right)$

${ }^{a}$ The XRE-I sequence motif is CACGCNA, and the XRE-II motif is CATGN ${ }_{6} \mathrm{C}$ T/A]TG as described in the Materials and Methods section.

${ }^{b} P$ value is given by the program FIMO to show the significance of the matching between putative XRE and the XRE motifs.

${ }^{c}$ Negative and positive values refer to the distance from the 5 '- or 3 '- ends of gene, respectively.

significant SNP-dependent difference in enrichment for the other three SNP/XRE fragments in ChIP assays (data not shown).

We selected LCLs for these initial studies of possible SNP-dependent CYP1A1 induction because of the availability of genome-wide SNP genotype information for these LCLs, which made it possible for us to select an adequate number of LCLs with the SNPs of interest for study. To determine whether the SNP-dependent CYP1A1 induction that we observed in LCLs might also exist in other cell lines, we created reporter gene constructs that included approximately $2.7 \mathrm{~kb}$ of the CYP1A1 promoter with WT and variant genotypes for the rs2470893 SNP (Fig. 2A). Sequences of the WT and variant reporter gene constructs were confirmed by DNA sequencing, which showed that they differed only in the single nucleotide that defined the rs2470893 SNP. These reporter gene constructs were then transfected into different cell lines to compare CYP1A1 transcriptional activities for WT and variant SNP genotypes in those cells.

The reporter gene constructs were first transfected into LCLs, and those cell lines were then treated with $3 \mathrm{MC}$ for 24 hours. LCLs transfected with the variant construct showed 1.5-fold higher CYP1A1 transcription activity than did cells transfected with the WT construct (Fig. 2B). The same constructs were then transfected into HepaRG cells, a cell line of hepatocyte origin that retains many of the characteristics of human hepatocytes, including expression of most of the cytochrome P450 enzymes (Guillouzo et al., 2007; Kanebratt and Andersson, 2008). The variant genotype construct once again showed significantly greater CYP1A1 transcriptional activity than did the WT construct (Fig. 2C). Since we had previously reported that AHR has a role in the regulation of kynurenine pathway genes in cells of central nervous system origin (Liu et al., 2018), the reporter gene constructs were also transfected into a neuroblastoma cell line, SK-N-BE(2), and a glioblastoma cell line, U-87 MG. A significant increase in transcriptional activity was observed in SK-N-BE(2) cells transfected with the variant construct compared with that for the WT construct (Fig. 2D), whereas no significant differences were observed in U-87 MG (Fig. 2E). These reporter gene assay results suggested that rs2470893 SNP-dependent CYP1A1 induction can be cell line-dependent.

A

CYP1A1

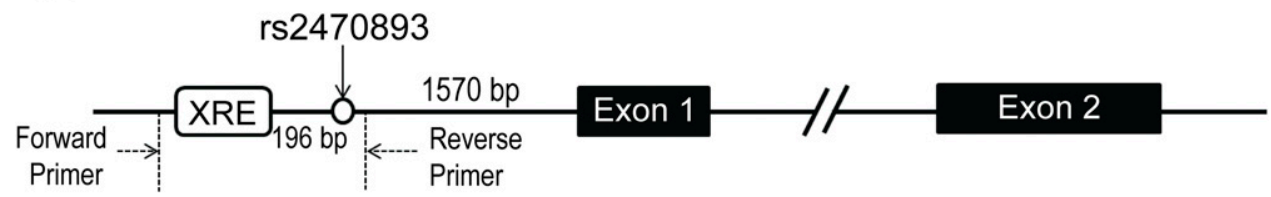

B

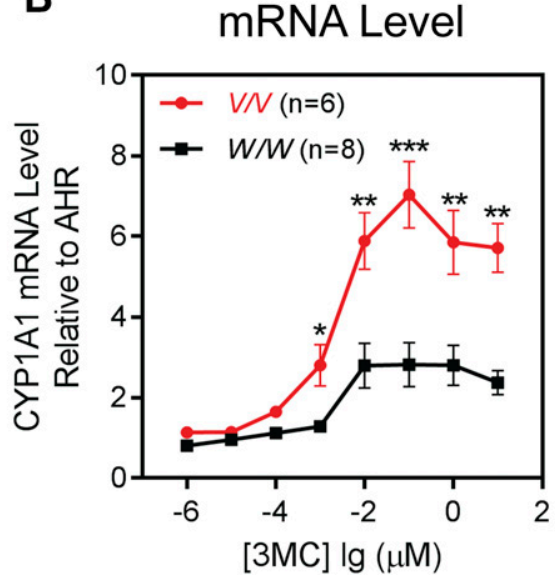

C

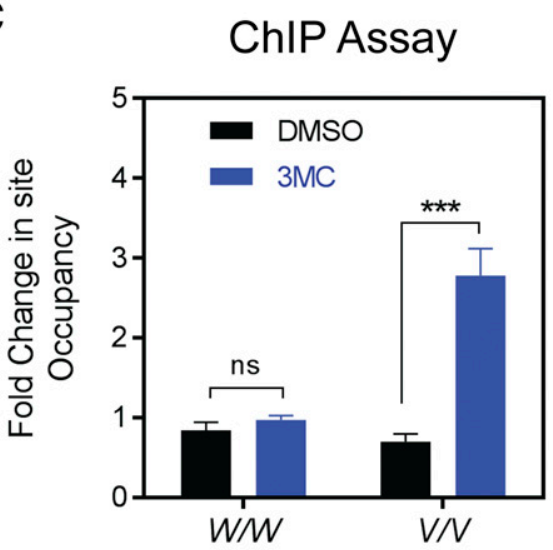

Fig. 1. (A) Schematic depiction of the location of the rs2470893 SNP and the nearby XRE at the 5'end of the CYPIAl gene. Annealing sites for the qPCR primers that were used in the ChIP assay are indicated. (B) After 24-hours of treatment with $3 \mathrm{MC}$ at a series of concentrations, LCLs homozygous for the rs $2470893 \mathrm{~V} / \mathrm{V}$ genotype $(n=6)$ showed significantly higher CYP1A1 mRNA expression than did LCLs homozygous for the $W / W$ genotype $(n=8)$. (C) ChIP assays performed with anti-AHR antibody showed a 3 -fold increase in AHR binding to the XRE 196 bp away from the $V / N$ genotype rs2470893 SNP compared with those homozygous for the $W / W$ genotype $(n=3)$. ${ }^{*} P<0.05$; ** $P<0.01$; *** $P<0.001$. Values shown are mean \pm S.E.M. 


\section{A CYP1A1 Reporter Gene Constructs}
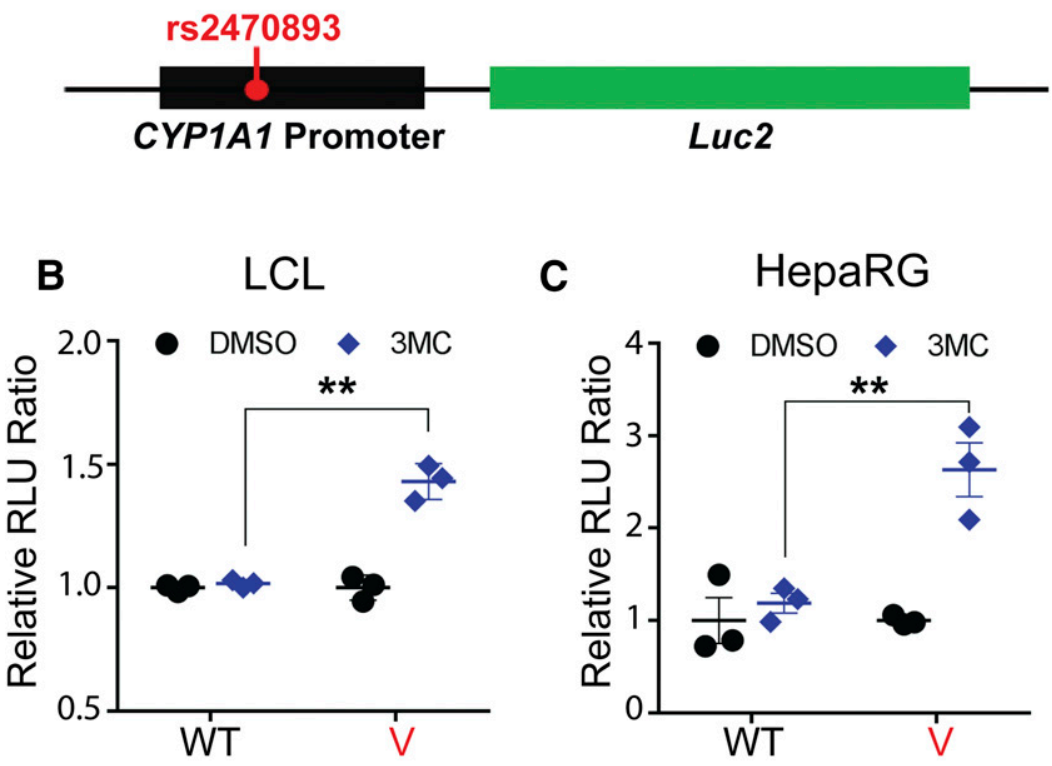

Fig. 2. Reporter gene assays for the $C Y P 1 A 1$ rs 2470893 SNP. (A) Structure of the CYPIAl promotor reporter gene construct within the basic pGL4 plasmid (Promega). The rs2470893 SNP was included in the CYPIAl promotor sequence that was subcloned upstream of the luciferase reporter gene, $L u c 2$. The plasmids contained either WT or variant sequences for the SNP within the CYP1Al promoter and were transfected into (B) LCLs, (C) HepaRG, (D) SK-NB-E(2), and (E) U-87 MG cells. Values shown for (B-E) are ratios of relative light units (RLUs) compared with the internal reference after $3 \mathrm{MC}$ or DMSO vehicle treatment. $* * P<0.01, \mathrm{~ns}$, not significant. Values shown are mean \pm S.E.M. for three determina-

D SK-N-BE(2)

E tions. Data for individual assays are shown in (B-E).
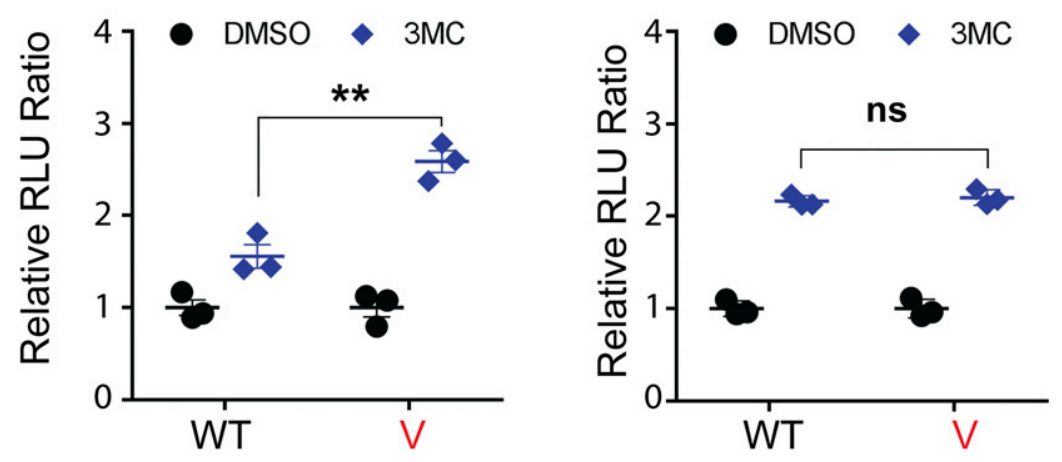

Identification of Possible rs2470893 SNP-Binding Proteins. To test potential mechanisms for the rs2470893 SNP-dependent CYP1A1 induction, EMSA was performed to identify potential AHR coregulator(s) that might also bind to the SNP region (Fig. 3A). Specifically, nuclear extract from LCLs treated with 3MC was incubated with 21 bp of DNA fragments with the SNP at middle of the sequence. DNA fragments with variant SNP genotype showed stronger binding to nuclear proteins than did those with the WT genotype (Fig. 3B). We then analyzed the EMSA bands by MS to identify proteins bound to the DNA fragments. "Blank" samples excised from the margins of gel were also submitted for MS analysis as controls. The proteins identified in each sample by MS are listed in Supplemental Table 7. Proteins present in "blank" samples and proteins that are commonly seen and, as a result, might represent nonspecific binding owing to their abundance in cells (Malovannaya et al., 2010; Hodge et al., 2013) were not pursued. Since MS identifies proteins based on the identity of peptide fragments, proteins homologous to those present in "blank" samples were also not included in subsequent studies. In addition, proteins encoded by pseudogenes and proteins that are not known to be present in the cell nucleus were excluded from further study. As a result, a total of 28 candidate proteins, including 19 proteins common in both WT and variant samples and nine proteins identified only in the variant sample, were selected for further study of their possible involvement in SNP-specific CYP1A1 induction (Fig. 3C).

To identify which of the rs2470893 SNP-binding proteins might influence CYP1A1 induction in an SNP-dependent fashion, we knocked down (KD) each of the 28 candidate genes encoding these proteins in an LCL with homozygous variant rs2470893 SNP genotype, followed by quantification of CYP1A1 mRNA expression after treatment with vehicle (DMSO) or 3MC. The effects of the KD of AHR were also determined as a positive control. CYP1A1 mRNA levels after the KD of AHR and the 28 candidate genes were then normalized to the negative control KD (neg. siRNA). After treatment with either DMSO or 3MC, CYP1A1 mRNA levels were dramatically decreased after the KD of AHR compared with the control KD (Fig. 3D). Meanwhile, KD of several of the candidate genes also resulted in a decrease in CYP1A1 mRNA levels (Fig. 3D). To determine which gene KD might have an effect similar to that of AHR KD, CYP1A1 mRNA expression levels after the KD of each gene by siRNA transfection were plotted with results for treatment with the DMSO vehicle on the $x$-axis and results for treatment with $3 \mathrm{MC}$ on the $y$-axis (Fig. 3E). Each dot in the plot shown graphically in Fig. 3E represents results for gene $\mathrm{KD}$ by siRNA 
A

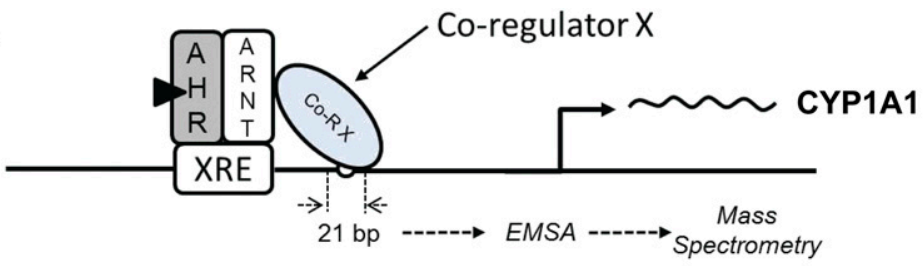

B

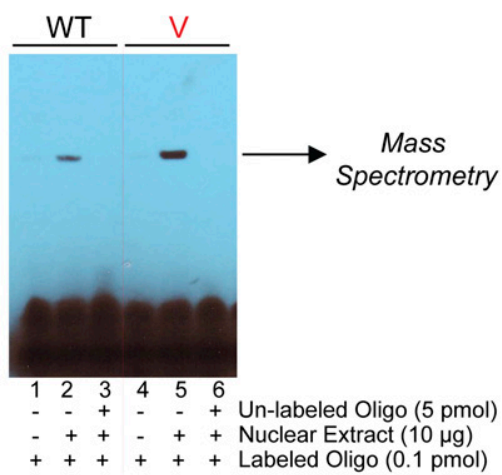

D

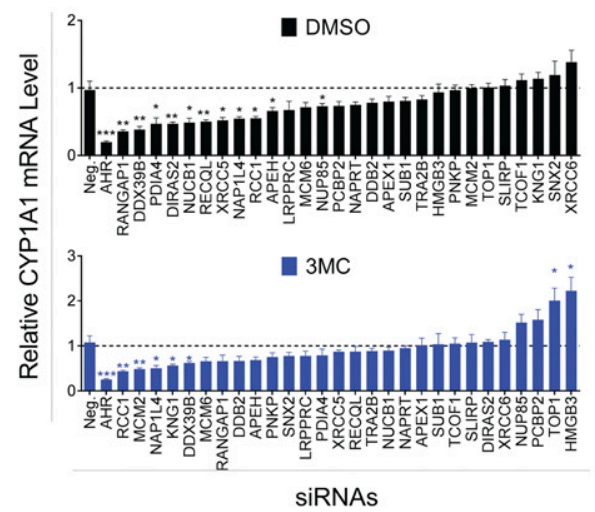

C

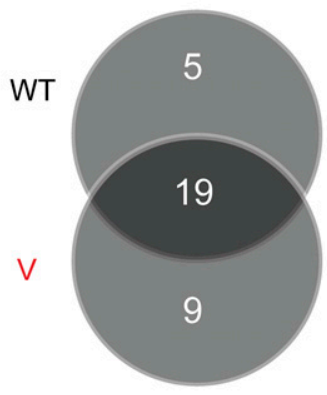

E

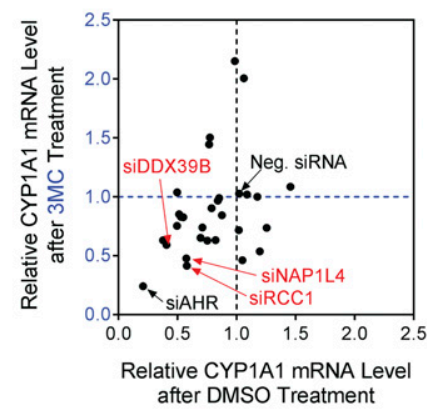

Fig. 3. (A) Schematic depiction of the hypothesis that coregulators might bind to the SNP and the AHR/ARNT complex to regulate CYP1A1 transcription. (B) Oligonucleotides $21 \mathrm{bp}$ long with the SNP in the middle were synthesized for EMSA assays, which showed that oligonucleotides with the variant genotype displayed greater LCL nuclear extract binding after 3MC treatment than did those with the WT genotype. The EMSA bands were sent for MS analysis to identify proteins that bound to the SNP DNA sequences. (C) Venn diagram for the MS data summary. A total of 28 proteins, including 19 that were present in both WT and variant bands and nine specific for the variant band, were selected for subsequent study. (D) Comparison of CYP1A1 mRNA expression levels after $\mathrm{KD}$ of 28 candidate proteins that had been identified by MS, together with data for the KD of AHR, followed by DMSO or 3MC treatment; $* P<0.05$; ** $P<0.01 ; * * * P<0.001$. Values shown are mean \pm S.E.M. for three determinations. (E) Correlations of ratios of CYP1A1 mRNA expression after DMSO or 3MC treatment indicated that KD of the RCC1, NAP1LA, and DDX39B genes (see red arrows) resulted in $C Y P 1 A 1$ ratios similar to those found after the $\mathrm{KD}$ of AHR. Dots represent mean values for three determinations. transfection, with its position in this figure dependent on CYP1A1 mRNA level after treatment with either DMSO and 3MC. The dot for AHR KD (siAHR) was closest to the origin (Fig. 3E), as anticipated, because CYP1A1 mRNA expression was dramatically decreased after the KD of AHR after treatment with the vehicle, DMSO, and 3MC. CYP1A1 mRNA levels after the KD of Nucleosome Assembly Protein 1 Like 4 (NAP1L4), Regulator of Chromosome Condensation 1 (RCC1), or DExD-Box Helicase 39B (DDX39B) were close to the position of results seen after AHR KD (Fig. 3E), raising the possibility that those three proteins might be involved in the AHR/ARNT transcriptional complex which binds near the rs2470893 SNP.

NAP1L4, RCC1, and DDX39B Contribute to rs2470893 SNPDependent CYP1A1 Induction. To determine whether NAP1L4, RCC1, and DDX39B proteins might contribute to SNP-dependent CYP1A1 induction, the KD of those three genes was repeated in LCLs with homozygous variant $(V / V)$ and WT $(W / W)$ rs 2470893 SNP genotypes, followed by CYP1A1 mRNA quantification. Although CYP1A1 mRNA expression was significantly increased after treatment with $3 \mathrm{MC}$ compared with DMSO vehicle treatment, KD of those three genes, as well as the KD of AHR, significantly decreased CYP1A1 induction compared with control KD in LCLs with a $V / V$ genotype (Fig. 4A, left). After plotting CYP1A1 mRNA levels after KD of those three candidate genes in LCLs homozygous for $V / V$ SNP genotypes with treatments that consisted of DMSO vehicle shown on the $x$-axis and $3 \mathrm{MC}$ treatment results shown on the $y$-axis, dots for KD of those three genes were once again located near the dot showing results for AHR KD (Fig. 4A, right); however, in LCLs homozygous for the $W / W$ rs 2470893 SNP genotype, KD of those three proteins did not change CYP1A1 mRNA levels induced by $3 \mathrm{MC}$ treatment (Fig. 4B, left). In that case, dots depicting the results for KD of those three genes were close to that for the nontargeting control $\mathrm{KD}$ (Fig. 4B, right), indicating that the NAP1L4, RCC1, and DDX39B proteins did not appear to significantly affect CYP1A1 induction in LCLs with $W / W$ genotypes for the rs2470893 SNP.

Using reporter gene constructs for WT and variant SNP genotypes for the rs2470893 SNP, we had demonstrated SNP-dependent CYP1A1 transcriptional activity in HepaRG cells (Fig. 2B). To determine whether NAP1L4, RCC1, and DDX39B might influence CYP1A1 induction in HepaRG cells, these reporter gene assays were performed once again after separate KD of each of those three genes. In the control KD group (neg. siRNA), CYP1A1 transcriptional activity was induced by $3 \mathrm{MC}$ treatment, and the activity was once again significantly increased in cells transfected with the variant reporter gene construct compared with the results for the WT construct (Supplemental Fig. 1A). As expected, $\mathrm{KD}$ of AHR (siAHR) repressed CYP1Al transcriptional activity for both WT and variant reporter gene constructs, and no significant 
A

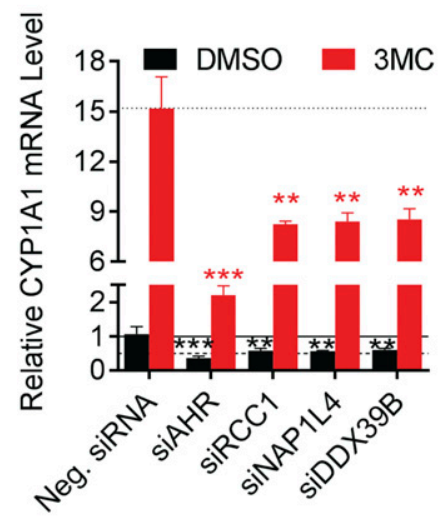

rs2470893 (V/N)

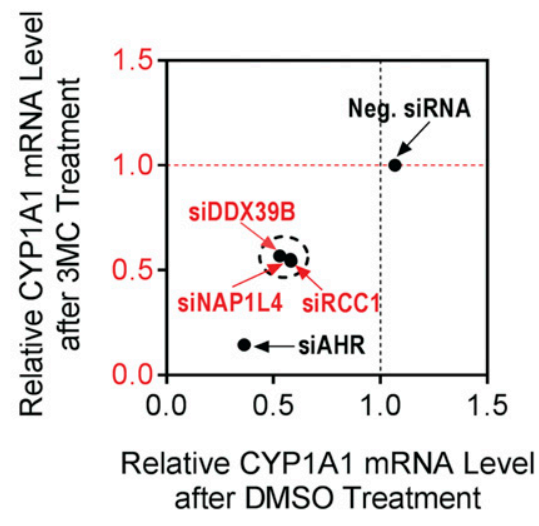

Fig. 4. (A, left) CYP1A1 induction by $3 \mathrm{MC}$ was decreased in LCLs with the rs2470893 $V / V$ genotype after the KD of RCC1, NAP1L4, or DDX39B. (A, right) Correlation of CYP1A1 mRNA levels after DMSO or 3MC treatment showing that CYP1A1 mRNA expression after the KD of RCC1, NAP1L4, or DDX39B was similar to results obtained after the KD of AHR in rs2470893 V/V LCLs. (B, left) CYP1A1 induction by $3 \mathrm{MC}$ was unchanged in LCLs with the rs2470893 $W / W$ genotype after the KD of RCC1, NAP1L4, or DDX39B, and (B, right), in contrast, CYP1A1 mRNA expression after the KD of RCC1, NAP1L4, or DDX39B was similar to data for the KD control in LCLs with the rs $2470893 W / W$ genotype. $* P<$ 0.05 ; $* * P<0.01 ; * * * P<0.001$. Values shown on the left are mean \pm S.E.M. for four determinations. Dots and squares shown on the right represent mean values for four determinations.
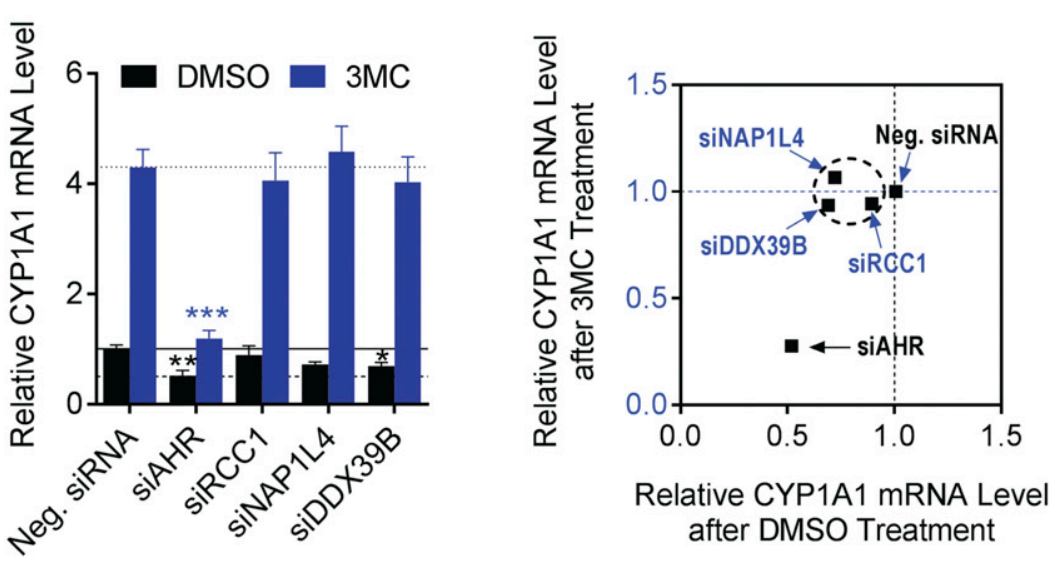

difference in 3MC-induced CYP1A1 transcriptional activity was found between WT and V reporter gene constructs (Supplemental Fig. 1A). Furthermore, compared with control KD, KD of NAP1L4, RCC1, or DDX39B in HepaRG cells also significantly repressed CYP1A1 transcriptional activity; as in the case of AHR KD, no differences in CYP1A1 transcriptional activity were observed between WT and variant reporter gene constructs (Supplemental Fig. 1A). Finally, AHR, RCC1, NAP1L4, and DDX39B mRNA levels were decreased significantly after specific siRNA transfections, as measured by qRT-PCR (Supplemental Fig. 1B). These results also indicated that NAP1L4, RCC1, and DDX39B might play a role in the SNP-dependent induction of CYP1A1 in HepaRG cells.

RCC1 and NAP1L4 Interact Physically with AHR. To determine whether NAP1L4, RCC1, or DDX39B might physically interact with the AHR transcriptional complex, coimmunoprecipitation (Co-IP) with anti-AHR antibody was performed using LCLs treated with 3MC. After precipitation with anti-AHR antibody, the samples were analyzed by Western blot analysis to determine whether NAP1L4, RCC1 or DDX39B might precipitate with AHR. As shown in Fig. 5A, RCC1 and NAP1L4 proteins were precipitated by the anti-AHR antibody, but DDX39B protein was not. To further confirm protein-protein interactions with AHR, "reverse" co-IP was performed using antibodies against NAP1L4, RCC1 and DDX39B, respectively, followed by detection performed by AHR precipitation. AHR was detected after precipitation with anti-RCC1 (Fig. 5B) and NAP1L4 (Fig. 5C) antibodies but not after precipitation with anti-DDX39B antibody (data not shown). These Co-IP studies confirmed that RCC1 and NAP1L4 could physically interact with AHR. When joined with the EMSA assay results, CYP1A1 mRNA expression results and the luciferase reporter gene assay results, these observations suggest that RCC1 and NAP1L4 proteins might interact with the rs2470893 SNP and enhance the binding of AHR to the nearby XRE, thus contributing to the regulation of CYP1A1 expression.

\section{Discussion}

Based on our previous observations with respect to the modulation of ER $\alpha$-ERE binding and downstream gene transcription (Ingle et al., 2013, 2016; Ho et al., 2016; Liu et al., 2017; Qin et al., 2017), in the present study, we set out to test the hypothesis that SNPs hundreds of bp distant from XREs might influence AHR-XRE binding and downstream gene expression. We chose to study $C Y P 1 A 1$, a prototypical AHR-regulated gene, by analyzing DNA sequences across and both up and downstream of the open reading frame for putative XREs and common SNPs that mapped $\pm 500 \mathrm{bp}$ of those XREs. We identified one SNP, rs2470893, located 5' of CYP1A1, that was associated with significant variation in AHR binding to a nearby (196 bp distant) XRE and with CYP1A1 transcription in LCLs (Fig. 1). This rs2470893 SNP not only influenced CYP1A1 transcriptional activity in LCLs but also appeared to be functionally significant in cell lines of different 

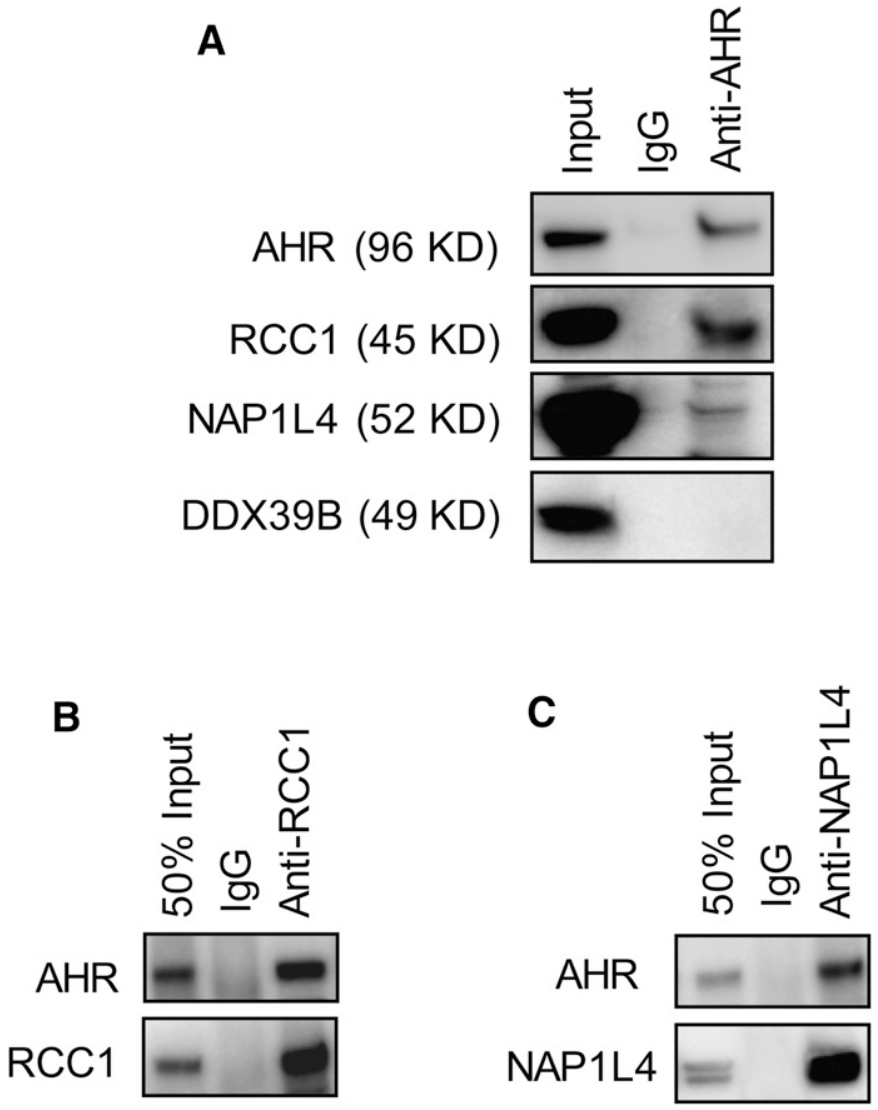

Fig. 5. (A) Co-immunoprecipitation (Co-IP) with anti-AHR antibody identified interactions between AHR and RCC1 and NAP1L4 in LCLs that were treated with 3MC but not for DDX39B. Reverse Co-IP with (B) anti-RCC1 and (C) antiNAP1L4 antibodies confirmed interactions between AHR and RCC1 and between AHR and NAP1L4. The data shown are representative of the results of two individual experiments.

tissue origins, including the hepatocyte-like HepaRG cells (Fig. 2). We then performed a series of studies to investigate possible molecular mechanisms for distant-SNP modulated AHR-XRE binding and gene transcription. Three proteins, NAP1L4, RCC1, and DDX39B were identified by MS after EMSA assay that could interact with DNA sequences that included the rs2470893 SNP and which were associated with AHR-dependent and SNP-dependent CYP1A1 induction. Two of those proteins also interacted physically with AHR, which indicates that they may be involved in the mechanism underlying distant-SNP modulated CYP1A1 transcription. Those findings provide, to our knowledge, the first example of SNPs that are near, but not in, an XRE and can regulate AHR-XRE binding and downstream gene transcription.

Because of its important role in the metabolism of environmental toxins and procarcinogens, CYP1A1 genetic polymorphisms have been studied for their possible association with the risk of the occurrence of cancer, but the results have often been contradictory. Those studies mainly focused on two common CYP1Al SNPs, rs4646903 (also known as $M s p I$ mutant or $C Y P 1 A 1 * 2 A$ ) and the rs 1048943 (Ile462Val) SNP; however, the molecular mechanism by which those two SNPs might function in the regulation of CYP1A1 activity remains unclear, which has hampered our understanding of the possible relationship between $C Y P 1 A 1$ genetic polymorphisms and clinical phenotypes. By systematically testing a novel mechanism by which SNPs at a distance from XREs might modulate AHR function and CYP1A1 induction, we have demonstrated that the $C Y P 1 A 1$ rs2470893 SNP variant genotype results in enhanced AHR binding to a nearby XRE, with increased CYP1A1 transcription after $3 \mathrm{MC}$ treatment. Our results fit with an observation reported previously that the rs2470893 SNP was significantly associated with circulating polychlorinated biphenyl 118 levels in a general elderly population $(n=1016)$, with the variant genotype associated with a decrease in PCB118 concentrations (Lind et al., 2014). PCBs are AHR agonists and are metabolized by CYP1A1. The decrease in PCB 118 plasma concentrations in subjects who carry the rs 2470893 variant genotype might be a consequence of enhanced induction of CYP1A1 expression.

The CYP1A1 and CYP1A2 genes are located "head to head"; as a result, they share the same 5 ' region on chromosome 15 . The rs 2470893 SNP is located between the two genes, approximately $1.5 \mathrm{~kb}$ upstream of CYP1A1 and $29.5 \mathrm{~kb}$ upstream of the CYP1A2 transcription start site. CYP1A2 is the major enzyme that metabolizes caffeine (Butler et al., 1989; Berthou et al., 1991). Several large-cohort GWAS studies for coffee consumption have identified rs2470893 as one of the "top hits" for association with that phenotype, with the variant genotype associated with less coffee consumption (Cornelis et al., 2011; Amin et al., 2012) and decreased caffeine metabolism (Cornelis et al., 2016). Caffeine was not found to induce CYP1A2 activity in human hepatocytes (Vaynshteyn and Jeong, 2012), but it did repress CYP1A1 expression in LCLs (Amin et al., 2012). It would be interesting to determine whether the rs2470893 variant genotype might enhance the caffeine effect on CYP1A2 repression and decrease caffeine metabolism; however, since CYP1A2 is not expressed in LCLs (data not shown), we were not able to study the possible effects of the SNP on CYP1A2 transcription in our cell line-based model system. Future studies using hepatocytes from individuals with different SNP genotypes might help to clarify the possible role of this SNP, or other genetically linked SNPs, in caffeine's effect on CYP1A2.

Functional genomic studies have largely been focused on genetic variation that maps to exons that alter the encoded protein amino acid sequence or within canonical splice sequences that alter RNA splicing; however, most GWAS top-hit SNP signals map to introns or intergenic regions, which indicates that noncoding SNPs play an important functional role, a role that often involves variation in transcription regulation (Battle et al., 2017; Weinshilboum and Wang, 2017; Wang et al., 2018). Gene transcription is often initiated by transcription factor binding to specific DNA sequence motifs, with the AHR/ARNT heterodimer binding to XREs, followed by recruitment of coregulators and RNA polymerase to form a transcriptional complex (Malovannaya et al., 2011). We have previously reported that proteins encoded by members of the testis specific Y-like (TSPYL) gene family were coregulators for the transcription of cytochrome P450s, including CYP3A4, CYP2C9, CYP2C19, CYP17A1, and CYP19A1 (Liu et al., 2013; Qin et al., 2018). Coregulators like members of the TSPYL gene family may, among other mechanisms, bind to specific DNA sequences, alter the nucleosome structure or recruit RNA polymerase, thus facilitating gene transcription (Qin et al., 2017). Therefore, changes in the binding of coregulators to DNA sequences as a result of SNPs that map hundreds of base pairs from response elements might well affect downstream gene transcription. In the present study, we identified NAP1L4 and RCC1 as possible AHR coregulators that interact with rs2470893 SNP sequences and influence CYP1A1 transcription in LCLs and in HepaRG cells. NAP1L4, also known as nucleosome assembly protein-2, is a histone chaperone protein that can transfer histones onto naked DNA templates (Rodriguez et al., 1997) and it functions within complexes with other proteins that are distinct from histones (Rodriguez et al., 2004). RCC1 is a 
chromatin-bound protein that binds directly with the nucleosome (Makde et al., 2010). Whether those two proteins regulate AHR transcriptional activity though interaction with nucleosomes needs to be determined in future studies. Finally, the DDX39B protein, which was shown to bind the rs2470893 SNP sequence and affect CYP1A1 transcriptional activity (Fig. 3, D and E), did not physically interact with AHR (Fig. 5A). Whether DDX39B might interact with other proteins recruited to the AHR-ARNT transcription complex needs to be determined.

In summary, by testing the hypothesis that SNPs distant from XRE motifs can modulate AHR transcriptional activity, we have demonstrated that the rs2470893 SNP modulates AHR-dependent CYP1A1 transcription. This "distant SNP" modulation of AHR transcriptional activity might be a mechanism that, in part, explains variation in the induction of CYP1A1 and, perhaps, other SNPs that influence the expression of other AHR-regulated genes. RNA sequencing and ChIP sequencing using LCLs and other genome data-rich cell lines might result in the discovery of additional SNPs distant from XREs that modulate AHR-dependent gene transcription.

\section{Authorship Contributions}

Participated in research design: Liu, Qin, Ray, Weinshilboum.

Conducted experiments: Liu, Qin, Kalari.

Contributed new reagents and analytical procedures: Liu, Qin, Wang, Weinshilboum.

Performed data analysis: Liu, Qin, Kalari, Weinshilboum.

Wrote or contributed to the writing of the manuscript: Liu, Weinshilboum.

\section{References}

Amin N, Byrne E, Johnson J, Chenevix-Trench G, Walter S, Nolte IM, Vink JM, Rawal R, Mangino M, Teumer A, et al.; kConFab Investigators (2012) Genome-wide association analysis of coffee drinking suggests association with CYP1A1/CYP1A2 and NRCAM. Mol Psychiatry 17:1116-1129.

Anttila S, Tuominen P, Hirvonen A, Nurminen M, Karjalainen A, Hankinson O, and Elovaara E (2001) CYP1A1 levels in lung tissue of tobacco smokers and polymorphisms of CYP1A1 and aromatic hydrocarbon receptor. Pharmacogenetics 11:501-509.

Battle A, Brown CD, Engelhardt BE, and Montgomery SB; GTEx Consortium; Laboratory, Data Analysis \&Coordinating Center (LDACC)—Analysis Working Group; Statistical Methods groups-Analysis Working Group; Enhancing GTEx (eGTEx) groups; NIH Common Fund; NIH/NCI; NIH/NHGRI; NIH/NIMH; NIH/NIDA; Biospecimen Collection Source Site-NDRI; Biospecimen Collection Source Site-RPCI; Biospecimen Core Resource-VARI; Brain Bank Repository-University of Miami Brain Endowment Bank; Leidos Biomedical-Project Management; ELSI Study; Genome Browser Data Integration \&Visualization-EBI; Genome Browser Data Integration \&Visualization-UCSC Genomics Institute, University of California Santa Cruz; Lead analysts; Laboratory, Data Analysis \&Coordinating Center (LDACC); NIH program management; Biospecimen collection; Pathology; eQTL manuscript working group (2017) Genetic effects on gene expression across human tissues. Nature 550:204-213.

Berthou F, Flinois JP, Ratanasavanh D, Beaune P, Riche C, and Guillouzo A (1991) Evidence for the involvement of several cytochromes P-450 in the first steps of caffeine metabolism by human liver microsomes. Drug Metab Dispos 19:561-567.

Butler MA, Iwasaki M, Guengerich FP, and Kadlubar FF (1989) Human cytochrome P-450PA (P-450IA2), the phenacetin O-deethylase, is primarily responsible for the hepatic 3-demethylation of caffeine and N-oxidation of carcinogenic arylamines. Proc Natl Acad Sci USA 86:7696-7700

Carey MF, Peterson CL, and Smale ST (2009) Chromatin immunoprecipitation (ChIP). Cold Spring Harb Protoc 2009:pdb prot5279.

Cornelis MC, Kacprowski T, Menni C, Gustafsson S, Pivin E, Adamski J, Artati A, Eap CB Ehret G, Friedrich N, et al.; Swiss Kidney Project on Genes in Hypertension (SKIPOGH) team (2016) Genome-wide association study of caffeine metabolites provides new insights to caffeine metabolism and dietary caffeine-consumption behavior. Hum Mol Genet 25: $5472-5482$.

Cornelis MC, Monda KL, Yu K, Paynter N, Azzato EM, Bennett SN, Berndt SI, Boerwinkle E, Chanock S, Chatterjee N, et al. (2011) Genome-wide meta-analysis identifies regions on $7 \mathrm{p} 21$ (AHR) and 15q24 (CYP1A2) as determinants of habitual caffeine consumption. PLoS Genet 7 e1002033.

Dahl JA and Collas P (2008) A rapid micro chromatin immunoprecipitation assay (microChIP). Nat Protoc 3:1032-1045.

Denison MS, Fisher JM, and Whitlock JP, Jr (1988) The DNA recognition site for the dioxin-Ah receptor complex. Nucleotide sequence and functional analysis. J Biol Chem 263: $17221-17224$.

Denison MS and Nagy SR (2003) Activation of the aryl hydrocarbon receptor by structurally diverse exogenous and endogenous chemicals. Annu Rev Pharmacol Toxicol 43: 309-334.

Guillouzo A, Corlu A, Aninat C, Glaise D, Morel F, and Guguen-Guillouzo C (2007) The human hepatoma HepaRG cells: a highly differentiated model for studies of liver metabolism and toxicity of xenobiotics. Chem Biol Interact 168:66-73.
Ho MF, Bongartz T, Liu M, Kalari KR, Goss PE, Shepherd LE, Goetz MP, Kubo M, Ingle JN, Wang L, et al. (2016) Estrogen, SNP-dependent chemokine expression and selective estrogen receptor modulator regulation. Mol Endocrinol 30:382-398.

Hodge K, Have ST, Hutton L, and Lamond AI (2013) Cleaning up the masses: exclusion lists to reduce contamination with HPLC-MS/MS. J Proteomics 88:92-103.

Ingle JN, Liu M, Wickerham DL, Schaid DJ, Wang L, Mushiroda T, Kubo M, Costantino JP, Vogel VG, Paik S, et al. (2013) Selective estrogen receptor modulators and pharmacogenomic variation in ZNF423 regulation of BRCA1 expression: individualized breast cancer prevention. Cancer Discov 3:812-825.

Ingle JN, Xie F, Ellis MJ, Goss PE, Shepherd LE, Chapman JW, Chen BE, Kubo M, Furukawa Y, Momozawa Y, et al. (2016) Genetic polymorphisms in the long noncoding RNA MIR2052HG offer a pharmacogenomic basis for the response of breast cancer patients to aromatase inhibitor therapy. Cancer Res 76:7012-7023.

Inoue H, Kiyohara C, Marugame T, Shinomiya S, Tsuji E, Handa K, Hayabuchi H, Onuma K, Hamada H, Koga H, et al. (2000) Cigarette smoking, CYP1A1 MspI and GSTM1 genotypes, and colorectal adenomas. Cancer Res 60:3749-3752.

Kanebratt KP and Andersson TB (2008) Evaluation of HepaRG cells as an in vitro model for human drug metabolism studies. Drug Metab Dispos 36:1444-1452.

Kawajiri K, Nakachi K, Imai K, Yoshii A, Shinoda N, and Watanabe J (1990) Identification of genetically high risk individuals to lung cancer by DNA polymorphisms of the cytochrome P450IA1 gene. FEBS Lett 263:131-133.

Kiyohara C, Nakanishi Y, Inutsuka S, Takayama K, Hara N, Motohiro A, Tanaka K, Kono S, and Hirohata T (1998) The relationship between CYP1A1 aryl hydrocarbon hydroxylase activity and lung cancer in a Japanese population. Pharmacogenetics $\mathbf{8}$ : 315-323.

Lind L, Penell J, Syvänen AC, Axelsson T, Ingelsson E, Morris AP, Lindgren C, Salihovic S, van Bavel B, and Lind PM (2014) Genetic variation in the CYP1A1 gene is related to circulating PCB118 levels in a population-based sample. Environ Res 133:135-140.

Liu D, Ho MF, Schaid DJ, Scherer SE, Kalari K, Liu M, Biernacka J, Yee V, Evans J, Carlson E, et al. (2017) Breast cancer chemoprevention pharmacogenomics: deep sequencing and functional genomics of the ZNF423 and CTSO genes. NPJ Breast Cancer 3:30.

Liu D, Ray B, Neavin DR, Zhang J, Athreya AP, Biernacka JM, Bobo WV, Hall-Flavin DK, Skime MK, Zhu H, et al. (2018) Beta-defensin 1, aryl hydrocarbon receptor and plasma kynurenine in major depressive disorder: metabolomics-informed genomics. Transl Psychiatry 8:10

Liu M, Ingle JN, Fridley BL, Buzdar AU, Robson ME, Kubo M, Wang L, Batzler A, Jenkins GD, Pietrzak TL, et al. (2013) TSPYL5 SNPs: association with plasma estradiol concentrations and aromatase expression. Mol Endocrinol 27:657-670.

Lo R and Matthews J (2012) High-resolution genome-wide mapping of AHR and ARNT binding sites by ChIP-Seq. Toxicol Sci 130:349-361.

Makde RD, England JR, Yennawar HP, and Tan S (2010) Structure of RCC1 chromatin factor bound to the nucleosome core particle. Nature 467:562-566.

Malovannaya A, Lanz RB, Jung SY, Bulynko Y, Le NT, Chan DW, Ding C, Shi Y, Yucer N, Krenciute G, et al. (2011) Analysis of the human endogenous coregulator complexome. Cell 145:787-799.

Malovannaya A, Li Y, Bulynko Y, Jung SY, Wang Y, Lanz RB, O'Malley BW, and Qin J (2010) Streamlined analysis schema for high-throughput identification of endogenous protein complexes. Proc Natl Acad Sci USA 107:2431-2436.

Mimura J and Fujii-Kuriyama Y (2003) Functional role of AhR in the expression of toxic effects by TCDD. Biochim Biophys Acta 1619:263-268.

Murray IA, Patterson AD, and Perdew GH (2014) Aryl hydrocarbon receptor ligands in cancer: friend and foe. Nat Rev Cancer 14:801-814.

Nelson JD, Denisenko O, and Bomsztyk K (2006) Protocol for the fast chromatin immunoprecipitation (ChIP) method. Nat Protoc 1:179-185.

Niu N, Qin Y, Fridley BL, Hou J, Kalari KR, Zhu M, Wu TY, Jenkins GD, Batzler A, and Wang L (2010) Radiation pharmacogenomics: a genome-wide association approach to identify radiation response biomarkers using human lymphoblastoid cell lines. Genome Res 20:1482-1492.

Persson I, Johansson I, and Ingelman-Sundberg M (1997) In vitro kinetics of two human CYP1A1 variant enzymes suggested to be associated with interindividual differences in cancer susceptibility. Biochem Biophys Res Commun 231:227-230.

Qin S, Ingle JN, Liu M, Yu J, Wickerham DL, Kubo M, Weinshilboum RM, and Wang L (2017) Calmodulin-like protein 3 is an estrogen receptor alpha coregulator for gene expression and drug response in a SNP, estrogen, and SERM-dependent fashion. Breast Cancer Res 19:95.

Qin S, Liu D, Kohli M, Wang L, Vedell PT, Hillman DW, Niu N, Yu J, Weinshilboum RM, and Wang L (2018) TSPYL family regulates CYP17A1 and CYP3A4 expression: potential mechanism contributing to abiraterone response in metastatic castration-resistant prostate cancer. Clin Pharmacol Ther 104:201-210.

Rannug A, Alexandrie AK, Persson I, and Ingelman-Sundberg M (1995) Genetic polymorphism of cytochromes P450 1A1, 2D6 and 2E1: regulation and toxicological significance. J Occup Environ Med 37:25-36.

Riddick DS, Huang Y, Harper PA, and Okey AB (1994) 2,3,7,8-Tetrachlorodibenzo-p-dioxin versus 3-methylcholanthrene: comparative studies of Ah receptor binding, transformation, and induction of CYP1A1. J Biol Chem 269:12118-12128.

Rodriguez P, Munroe D, Prawitt D, Chu LL, Bric E, Kim J, Reid LH, Davies C, Nakagama H, Loebbert R, et al. (1997) Functional characterization of human nucleosome assembly protein-2 (NAP1L4) suggests a role as a histone chaperone. Genomics 44 253-265.

Rodriguez P, Ruiz MT, Price GB, and Zannis-Hadjopoulos M (2004) NAP-2 is part of multiprotein complexes in HeLa cells. $J$ Cell Biochem 93:398-408.

Saleh A, Alvarez-Venegas R, and Avramova Z (2008) An efficient chromatin immunoprecipitation (ChIP) protocol for studying histone modifications in Arabidopsis plants. Nat Protoc 3: $1018-1025$.

Smart J and Daly AK (2000) Variation in induced CYP1A1 levels: relationship to CYP1A1, Ah receptor and GSTM1 polymorphisms. Pharmacogenetics 10:11-24.

Smith GB, Harper PA, Wong JM, Lam MS, Reid KR, Petsikas D, and Massey TE (2001) Human lung microsomal cytochrome P4501A1 (CYP1A1) activities: impact of smoking status and CYP1A1, aryl hydrocarbon receptor, and glutathione S-transferase M1 genetic polymorphisms. Cancer Epidemiol Biomarkers Prev 10:839-853. 
Sogawa K, Numayama-Tsuruta K, Takahashi T, Matsushita N, Miura C, Nikawa J, Gotoh O, Kikuchi Y, and Fujii-Kuriyama Y (2004) A novel induction mechanism of the rat CYP1A2 gene mediated by $\mathrm{Ah}$ receptor-Arnt heterodimer. Biochem Biophys Res Commun 318 $746-755$

Spurr NK, Gough AC, Stevenson K, and Wolf CR (1987) Msp-1 polymorphism detected with a cDNA probe for the P-450 I family on chromosome 15 . Nucleic Acids Res 15: 5901.

Stücker I, Jacquet M, de Waziers I, Cénée S, Beaune P, Kremers P, and Hémon D (2000) Relation between inducibility of CYP1A1, GSTM1 and lung cancer in a French population. Pharmacogenetics 10:617-627.

Vaynshteyn D and Jeong H (2012) Caffeine induces CYP1A2 expression in rat hepatocytes but not in human hepatocytes. Drug Metab Lett 6:116-119.

Wang L, Ingle J, and Weinshilboum R (2018) Pharmacogenomic discovery to function and mechanism: breast cancer as a case study. Clin Pharmacol Ther 103:243-252.
Weinshilboum RM and Wang L (2017) Pharmacogenomics: precision medicine and drug response. Mayo Clin Proc 92:1711-1722.

Yu J, Qin B, Moyer AM, Nowsheen S, Liu T, Qin S, Zhuang Y, Liu D, Lu SW, Kalari KR, et al. (2018) DNA methyltransferase expression in triple-negative breast cancer predicts sensitivity to decitabine. J Clin Invest 128:2376-2388.

Zhang ZY, Fasco MJ, Huang L, Guengerich FP, and Kaminsky LS (1996) Characterization of purified human recombinant cytochrome P4501A1-Ile462 and -Val462: assessment of a role for the rare allele in carcinogenesis. Cancer Res 56:3926-3933.

Address correspondence to: Dr. Richard M. Weinshilboum, Mayo Clinic, 200 First Street SW, Rochester, MN 55905. E-mail: weinshilboum.richard@mayo.edu 